\title{
LINEARLY STABLE SUBHARMONIC ORBITS IN STRONGLY MONOTONE TIME-PERIODIC DYNAMICAL SYSTEMS
}

\author{
PETER TAKÁČ
}

(Communicated by Kenneth R. Meyer)

\begin{abstract}
We construct two simple examples of strongly monotone timeperiodic dynamical systems (of period $\tau>0$ ) possessing linearly stable subharmonic orbits of period $n \tau$ for any integer $n \geq 2$. The first example is an irreducible cooperative system of four ODE's that models positive feedback. The second example is a one-dimensional reaction-diffusion PDE with periodic boundary conditions. Our construction employs Chebyshev's polynomials.
\end{abstract}

\section{INTRODUCTION}

A very interesting phenomenon in a generic strongly monotone, autonomous dynamical system (with continuous time) is convergence to an equilibrium (or to a set of equilibria) for "almost every" relatively compact positive semiorbit, cf. Hirsch [10, 11, 13], Matano [15], Poláčik [17], Smith and Thieme [21, 22], and Takáč [25]. Typical examples of such dynamical systems are irreducible cooperative systems of ordinary differential equations and (irreducible cooperative systems of) reaction-diffusion equations where these equations are autonomous. Recently there have been several attempts to show similar convergence properties for strongly monotone systems of time-periodic (nonautonomous) equations by imposing additional hypotheses on these systems, such as Ljapunov stability of all equilibria (i.e., fixed points of the period map) or sublinear nonlinearities, cf. Alikakos and Hess [1], Alikakos, Hess, and Matano [2], Chen and Matano [5], Dancer and Hess [6], Hale and Somolinos [8], Hess and Weinberger [9], Hirsch [12], Smith [19], and Takáč [23, 24, 26, 27]. These articles study the period map $T$ associated with a given time-periodic system of equations, together with the discrete-time dynamical system $\left\{T^{n}: n \in \mathbb{Z}_{+}\right\}$generated by $T$ in a suitable underlying space $X$. Simple examples of strongly monotone discrete-time dynamical systems exhibiting nontrivial linearly stable cycles (and unstable equilibria) have been constructed in Dancer and Hess [6, §2] and Takáč [27, Example 4.4]. Until now no examples of such cycles have been known for

Received by the editors June 27, 1990.

1991 Mathematics Subject Classification. Primary 34C35, 35B40; Secondary 35K55, 47H07.

Key words and phrases. Positive feedback system, reaction-diffusion equation, strongly monotone mapping, period map, linearly stable cycle, Chebyshev's polynomial.

This research was supported in part by the National Science Foundation under the grant DMS8802646 and by the Vanderbilt University Research Council. 
the period map $T$ of a strongly monotone time-periodic system of equations with continuous time. In fact, Takáč [27] has ruled out this possibility in the case of a (multi-dimensional) time-periodic reaction-diffusion equation (or an irreducible cooperative system of two such equations) with (spatially) periodic boundary conditions assuming that both the diffusivity and the reaction function are spatially homogeneous. Namely, this assumption forces also the spatial homogeneity of every Ljapunov stable time-periodic solution. In addition, Chen and Matano [5] have ruled out the existence of any (stable or unstable) cycles by proving a convergence theorem in the case of a one-dimensional time-periodic reaction-diffusion equation with Dirichlet, Neumann, or periodic boundary conditions assuming (as above) spatially homogeneous diffusivity and reaction function. Similarly, Hale and Somolinos [8] proved a convergence theorem in the case of a time-periodic irreducible cooperative system of two ODE's.

In this article we construct two simple examples of strongly monotone timeperiodic dynamical systems of period $\tau>0$ possessing linearly stable subharmonic orbits of period $n \tau$ for any integer $n \geq 2$. Equivalently, the corresponding period map $T$ has a linearly stable $n$-cycle. Our first example $(\S 2)$ is an irreducible cooperative system of four ODE's that models single loop positive feedback. Our second example $(\S 3)$ is a one-dimensional rection-diffusion PDE (containing a transport term) with periodic boundary conditions. The nonlinearities in these two examples involve $n$th order Chebyshev's polynomials $T_{n}(u)$ (cf. Lebedev [14]) since they have the following property:

The function $g_{n}(\xi, u)=\cos (n \xi)-T_{n}(u)$ of $(\xi, u) \in \mathbb{R} \times \mathbb{R}$ defined for every fixed $n \in \mathbb{N}$ is $(2 \pi / n)$-periodic in the $\xi$-variable and satisfies $g_{n}(\xi, \cos \xi)=0$, whereas $\cos \xi$ is only $2 \pi$-periodic.

Finally, in $\S 4$ we present additional positive feedback-type (time-periodic) systems of ODE's where numerical computations strongly suggest (but do not prove) the presence of linearly stable subharmonic orbits.

\section{Preliminaries}

Throughout the article we use the following standard concepts and notation.

$X$ denotes a strongly ordered separable (real) Banach space, i.e., $X$ is a separable Banach space over $\mathbb{R}$ with a closed (partial) order relation " $\leq$ " in $X \times X$ such that the positive cone $X_{+}=\{x \in X: x \geq 0\}$ in $X$ has nonempty interior denoted by $\operatorname{Int}\left(X_{+}\right)$. We write $x<y$ to express $x \leq y$ and $x \neq y$ in $X$, whereas $x \ll y$ means $y-x \in \operatorname{Int}\left(X_{+}\right)$for $x, y \in X$.

$U \equiv\{U(t, s): t \geq s \geq 0\}$ denotes a time-periodic dynamical system (or time-periodic process) in $X$ of period $\tau>0$, which is assumed to be strongly monotone: $x<y$ in $X$ implies $U(t, s) x \ll U(t, s) y$ whenever $t>s \geq 0$. The corresponding period map $T: X \rightarrow X$ is defined by $T=U(\tau, 0)$. We recall that a nonautonomous dynamical system (or process) is a continuous mapping $U$ from $\left\{(t, s) \in \mathbb{R}^{2}: t \geq s \geq 0\right\} \times X$ into $X$ satisfying $U(t, t) x=x$ and $U(t, r) x=U(t, s) U(s, r) x$ for all $t \geq s \geq r \geq 0$ and $x \in X$. It is called timeperiodic of period $\tau>0$ if also $U(t+\tau, s+\tau) x=U(t, s) x$ for all $t \geq s \geq 0$ and $x \in X$.

We use the discrete-time dynamical system $\left\{T^{n}: n \in \mathbb{Z}_{+}\right\}$in $X$ to introduce the following standard concepts.

The discrete-time positive semiorbit (briefly, orbit) of any $x \in X$ is defined 
by $\mathscr{O}^{+}(x)=\left\{T^{n} x: n \in \mathbb{Z}_{+}\right\}$, and the $\omega$-limit set of $x$ is defined by $\omega(x)=$ $\left\{y \in X: T^{n_{k}} x \rightarrow y \quad(k \rightarrow \infty)\right.$ for some sequence $n_{k} \rightarrow \infty$ in $\left.\mathbb{Z}_{+}\right\}$. Notice that if $\mathscr{O}^{+}(x)$ is relatively compact in $X$, then $\omega(x) \neq \varnothing$. The reader is referred to Hale [7, Chapter 2] for additional concepts such as global attractor, etc.

Analogously, the continuous-time positive semiorbit (shortly, continuous orbit) of any $x \in X$ is defined by $\gamma^{+}(x)=\left\{U(t, 0) x: t \in \mathbb{R}_{+}\right\}$. We say that $\gamma^{+}(x)$ is a subharmonic orbit of period $n \tau$, for some integer $n \geq 2$, iff $\mathscr{O}^{+}(x)$ is an $n$-cycle, i.e., $T^{n} x=x$ and $\mathscr{O}^{+}(x)$ has precisely $n$ distinct elements. Such a subharmonic orbit $\gamma^{+}(x)$ is called linearly stable iff the cycle $\mathscr{O}^{+}(x)$ is linearly stable, i.e., the mapping $T^{n}: X \rightarrow X$ is Fréchet differentiable at its fixed point $x \in X$ and the corresponding Fréchet derivative $D\left(T^{n}\right)(x)$ has spectral radius $\operatorname{spr}\left(D\left(T^{n}\right)(x)\right)<1$.

Finally, given any integer $n \geq 0$, the $n$th Chebyshev's polynomial $T_{n}(u)$ is uniquely determined by $T_{n}(u)=\cos (n \arccos u)$ for $u \in[-1,1]$. For $n \geq 1$ we define an auxiliary function $f: \mathbb{R} \times \mathbb{R} \rightarrow \mathbb{R}$ by $f(\xi, u) \equiv$ $\left(\cos (n \xi)-T_{n}(u)\right) T_{n}^{\prime}(u)$, which is a polynomial of order $2 n-1$ in $u$ with the leading coefficient $-n \cdot 2^{2(n-1)}$. We set

$$
\varphi(\xi) \equiv-\frac{\partial f}{\partial u}(\xi, \cos \xi)=\left(T_{n}^{\prime}(\cos \xi)\right)^{2}=\left(n \frac{\sin (n \xi)}{\sin \xi}\right)^{2}, \quad \xi \in \mathbb{R}
$$

\section{A SYSTEM OF FOUR ODE'S}

Our first example is the following single loop positive feedback system for the unknown vector $u=u(t) \equiv\left(u_{1}, u_{2}, u_{3}, u_{4}\right) \in X \equiv \mathbb{R}^{4}$ depending on time $t \in \mathbb{R}_{+}$:

$$
\begin{aligned}
\frac{d u_{1}}{d t} & =u_{4}+\alpha f\left(t, u_{1}\right), & \frac{d u_{2}}{d t} & =u_{1}+\alpha f\left(t-\frac{\pi}{2}, u_{2}\right), \\
\frac{d u_{3}}{d t} & =u_{2}+\alpha f\left(t+\pi, u_{3}\right), & \frac{d u_{4}}{d t} & =u_{3}+\alpha f\left(t+\frac{\pi}{2}, u_{4}\right),
\end{aligned}
$$

with a given initial value $u(0) \in \mathbb{R}^{4}$. Here $\alpha>0$ is a sufficiently large constant to be determined later, and the function $f$ has been defined in $\S 1$. The Euclidean space $\mathbb{R}^{4}$ is endowed with the natural coordinatewise ordering. This system in $\mathbb{R}^{4}$ is $(2 \pi / n)$-periodic, cooperative, and irreducible, and hence, it is strongly monotone by the Müller-Kamke theorem, cf. Hirsch [10, 11], Selgrade [18], or Smith [20]. The nonlinearity $f(\xi, u)=-n \cdot 2^{2(n-1)} u^{2 n-1}+\cdots$ guarantees the existence and uniqueness of a classical $C^{\infty}$-solution $u(t)$ of (1) and the existence of a global attractor as well. Hence, (1) generates a strongly monotone $(2 \pi / n)$-periodic dynamical system $U(t, s), t \geq s \geq 0$, in $\mathbb{R}^{4}$ that is determined by $u(t)=U(t, s) u(s), t \geq s \geq 0$, for an arbitrary initial value $u(s) \in \mathbb{R}^{4}$. It is obvious that the time-dependent vector

$$
\tilde{u}(t)=\left(\cos t, \cos \left(t-\frac{\pi}{2}\right), \cos (t+\pi), \cos \left(t+\frac{\pi}{2}\right)\right)=(\cos t, \sin t,-\cos t,-\sin t)
$$

is a solution of (1). Furthermore, system $(1)$ is $(2 \pi / n)$-periodic in the time variable $t$ whereas $\tilde{u}(t)$ is only $2 \pi$-periodic. We claim the following result:

Proposition 2.1. Let $n \geq 2$ and $\tau=2 \pi / n$. Then there exists a constant $\alpha_{n}>0$ depending only on $n$ such that $\tilde{u}(t)$ is a linearly stable subharmonic orbit of period $n \tau=2 \pi$ whenever $\alpha>\alpha_{n}$. 
Proof. It is well known that the linear stability of $\tilde{u}(t)$ follows from that of the zero solution of the linearization of system (1) about $\tilde{u}(t)$. This linearization has the following form with the unknown vector $v=v(t) \equiv\left(v_{1}, v_{2}, v_{3}, v_{4}\right) \in$ $\mathbb{R}^{4}$ :

$$
\begin{array}{llrl}
\frac{d v_{1}}{d t} & =v_{4}-\alpha \varphi(t) v_{1}, & \frac{d v_{2}}{d t} & =v_{1}-\alpha \varphi\left(t-\frac{\pi}{2}\right) v_{2}, \\
\frac{d v_{3}}{d t} & =v_{2}-\alpha \varphi(t+\pi) v_{3}, & \frac{d v_{4}}{d t} & =v_{3}-\alpha \varphi\left(t+\frac{\pi}{2}\right) v_{4} .
\end{array}
$$

Also system (2) is cooperative and irreducible, and consequently, strongly monotone. It is only $\pi$-periodic. We set

$$
\psi(t)=\min \left\{\varphi(t), \varphi\left(t-\frac{\pi}{2}\right), \varphi(t+\pi), \varphi\left(t+\frac{\pi}{2}\right)\right\}, \quad t \in \mathbb{R} .
$$

Next we consider the following majorization of system (2) with the unknown vector $w=w(t) \equiv\left(w_{1}, w_{2}, w_{3}, w_{4}\right) \in \mathbb{R}^{4}$ :

$$
\begin{array}{llrl}
\frac{d w_{1}}{d t} & =w_{4}-\alpha \psi(t) w_{1}, & & \frac{d w_{2}}{d t}=w_{1}-\alpha \psi(t) w_{2}, \\
\frac{d w_{3}}{d t}=w_{2}-\alpha \psi(t) w_{3}, & \frac{d w_{4}}{d t}=w_{3}-\alpha \psi(t) w_{4} .
\end{array}
$$

It follows from the Perron-Frobenius theory combined with the Müller-Kamke theorem that the linear stability of the zero solution of system (3) implies the same property for system (2). Namely, if $\left|v_{i}(s)\right| \leq w_{i}(s), i=1,2,3,4$, for some $s \geq 0$, then also $\left|v_{i}(t)\right| \leq w_{i}(t), i=1,2,3,4$, for all $t \geq s$. We set $A=\frac{1}{\pi} \int_{0}^{\pi} \psi(t) d t \quad(>0)$ and

$$
x(t)=w(t) \exp \left(\int_{0}^{t} \psi(s) d s-A t\right),
$$

where $\int_{0}^{t} \psi(s) d s-A t$ is a $\pi$-periodic function of $t \in \mathbb{R}$. Then $x(t)$ is a solution of the following autonomous system:

$$
\begin{array}{llrl}
\frac{d x_{1}}{d t} & =x_{4}-\alpha A x_{1}, & & \frac{d x_{2}}{d t}=x_{1}-\alpha A x_{2}, \\
\frac{d x_{3}}{d t}=x_{2}-\alpha A x_{3}, & \frac{d x_{4}}{d t}=x_{3}-\alpha A x_{4} .
\end{array}
$$

The zero solution of this system is linearly stable iff $\alpha A>1$, and so we may choose $\alpha_{n}=1 / A$. The proposition has been proved.

\section{A REACTION-DIFFUSION PDE}

Let $X \equiv C(\mathbb{R} / 2 \pi \mathbb{Z})$ denote the Banach space of all $2 \pi$-periodic continuous functions $f: \mathbb{R} \rightarrow \mathbb{R}$ endowed with the maximum norm $\|f\|_{\infty}=\max _{x \in \mathbb{R}}|f(x)|$ and the pointwise ordering. Our second example is the following reactiondiffusion equation (with transport) for the unknown vector-valued function $u=$ $u(t) \equiv u(\cdot, t) \in C(\mathbb{R} / 2 \pi \mathbb{Z})$ depending on time $t \in \mathbb{R}_{+}$, where "." stands for the space variable $x \in \mathbb{R}$ :

$$
\frac{\partial u}{\partial t}=d\left(\frac{\partial^{2} u}{\partial x^{2}}+u\right)-\frac{1}{n} \frac{\partial u}{\partial x}+\alpha f\left(x-\frac{t}{n}, u\right), \quad x \in \mathbb{R}, t>0
$$


with a given initial value $u(0) \in C(\mathbb{R} / 2 \pi \mathbb{Z})$. Here $d>0$ is an arbitrary diffusivity constant, $\alpha>0$ is a sufficiently large constant to be determined later, and the function $f$ was defined in $\S 1$. This equation in $C(\mathbb{R} / 2 \pi \mathbb{Z})$ is $2 \pi$-periodic and satisfies the strong maximum principle for parabolic equations (cf. Hirsch $[13, \S 5])$. The nonlinearity $f(\xi, u)=-n \cdot 2^{2(n-1)} u^{2 n-1}+\cdots$ guarantees the existence and uniqueness of a classical $C^{\infty}$-solution $u(x, t)$ of $(5)$ and the existence of a global attractor as well by the smoothing property of parabolic equations. Hence, $(5)$ generates a strongly monotone $2 \pi$-periodic dynamical system $U(t, s), t \geq s \geq 0$, in $C(\mathbb{R} / 2 \pi \mathbb{Z})$ that is determined by $u(t)=U(t, s) u(s)$, $t \geq s \geq 0$, for an arbitrary initial value $u(s) \in C(\mathbb{R} / 2 \pi \mathbb{Z}$ ) (cf. Amann [3, $\S 15$; $4, \S \S 2,3])$. It is obvious that the function $\tilde{u}(x, t)=\cos (x-t / n)$ is a solution of (5). Furthermore, equation (5) is $2 \pi$-periodic in the time variable $t$ whereas $\tilde{u}(x, t)$ is only $2 n \pi$-periodic in $t$. We claim the following result:

Proposition 3.1. Let $n \geq 2, d>0$, and $\tau=2 \pi$. Then there exists a constant $\alpha_{n}>0$ depending only on $n$ and $d$ such that $\tilde{u}(\cdot, t)$ is a linearly stable subharmonic orbit of period $n \tau=2 n \pi$ whenever $\alpha>\alpha_{n}$.

Proof. Similarly as in (1), the linear stability of $\tilde{u}(\cdot, t)$ follows from that of the zero solution of the linearization of equation $(5)$ about $\tilde{u}(\cdot, t)$. This linearization has the following form with the unknown function $v=v(x, t)$ :

$$
\frac{\partial v}{\partial t}=d\left(\frac{\partial^{2} v}{\partial x^{2}}+v\right)-\frac{1}{n} \frac{\partial v}{\partial x}-\alpha \varphi\left(x-\frac{t}{n}\right) v, \quad x \in \mathbb{R}, t>0 .
$$

Also equation (6) satisfies the strong maximum principle, and consequently, it generates a strongly monotone $n \pi$-periodic dynamical system in $C(\mathbb{R} / 2 \pi \mathbb{Z})$. We set $w(x, t)=v\left(x+\frac{t}{n}, t\right)$, whence $v(x, t)=w\left(x-\frac{t}{n}, t\right)$. Then $w=$ $w(t) \equiv w(\cdot, t) \in C(\mathbb{R} / 2 \pi \mathbb{Z})$ is a solution of the autonomous equation

$$
\frac{\partial w}{\partial t}=d\left(\frac{\partial^{2} w}{\partial x^{2}}+w\right)-\alpha \varphi(x) w, \quad x \in \mathbb{R}, t>0
$$

It is obvious that the zero solution of equation (6) is linearly stable iff the same property holds for equation (7).

Let $\mathscr{L} \equiv \mathscr{L}_{\alpha}: L^{2}(-\pi, \pi) \rightarrow L^{2}(-\pi, \pi)$ denote the selfadjoint linear operator defined by

$$
(\mathscr{L} w)(x)=\frac{\partial^{2} w}{\partial x^{2}}+w-\frac{\alpha}{d} \varphi(x) w, \quad x \in(-\pi, \pi),
$$

for each $w \in W^{2,2}(-\pi, \pi)$ satisfying $u(-\pi)=u(\pi)$ and $u^{\prime}(-\pi)=u^{\prime}(\pi)$ in the sense of traces. Since $\mathscr{L}$ is selfadjoint with compact resolvent, its entire spectrum lies in $\mathbb{R}$ and consists of a discrete set of eigenvalues only. Then the zero solution of (7) is linearly stable iff the largest eigenvalue $\lambda_{1} \equiv \lambda_{1, \alpha}$ of $\mathscr{L}$ is $<0$. By Nussbaum [16, $\S$ ], the largest eigenvalue $\lambda_{1}$ of $\mathscr{L}$ is simple with a strictly positive eigenfunction $w_{1} \equiv w_{1, \alpha} \gg 0$ normalized by $\int_{-\pi}^{\pi} w_{1}^{2}=1$, and also

$$
1-\lambda_{1}=\int_{-\pi}^{\pi}\left(w_{1}^{\prime}\right)^{2} d x+\frac{\alpha}{d} \int_{-\pi}^{\pi} w_{1}^{2} \varphi(x) d x
$$

By the strong maximum principle for elliptic equations, $\lambda_{1}$ is a strictly decreasing function of $\alpha \geq 0$. Consequently, it suffices to show $\lambda_{1, \alpha_{n}} \leq 0$ for some $\alpha_{n} \geq 0$. Indeed, we will prove $\lambda_{1} \searrow-\infty$ as $\alpha \nearrow \infty$. 
Suppose not; then $\lambda_{1} \searrow \hat{\lambda}_{1}>-\infty$ as $\alpha / \infty$. Consequently, (8) shows that the net $\left\{w_{1, \alpha}\right\}_{\alpha \in(0, \infty)}$ is bounded in $W^{1,2}(-\pi, \pi)$, and hence, relatively compact in $C(\mathbb{R} / 2 \pi \mathbb{Z}) \hookrightarrow L^{2}(-\pi, \pi)$. Furthermore, given any $\bar{\alpha}>0$ and $\alpha \geq \bar{\alpha},(8)$ entails

$$
1-\hat{\lambda}_{1} \geq \frac{\bar{\alpha}}{d} \int_{-\pi}^{\pi} w_{1, \alpha}^{2} \varphi(x) d x .
$$

Thus, we can extract a subsequence $\alpha_{k} \nearrow \infty$ such that $w_{1, \alpha_{k}} \rightarrow \widehat{w}_{1} \in C(\mathbb{R} / 2 \pi \mathbb{Z})$ in $C(\mathbb{R} / 2 \pi \mathbb{Z})$ as $k \nearrow \infty$. Then $\int_{-\pi}^{\pi} \hat{w}_{1}^{2}=1$, and by $(9)$,

$$
1-\hat{\lambda}_{1} \geq \frac{\bar{\alpha}}{d} \int_{-\pi}^{\pi} \hat{w}_{1}^{2} \varphi(x) d x
$$

holds for every $\bar{\alpha}>0$. Letting $\bar{\alpha} \rightarrow \infty$ we arrive at $\int_{-\pi}^{\pi} \hat{w}_{1}^{2} \varphi(x) d x=0$, which forces $\hat{w}_{1}=0$ in $C(\mathbb{R} / 2 \pi \mathbb{Z})$, thus contradicting $\int_{-\pi}^{\pi} \hat{w}_{1}^{2}=1$. We have proved $\lambda_{1} \searrow-\infty$ as $\alpha \nearrow \infty$, and also our proposition.

\section{REMARKS}

Another $2 \pi$-periodic positive feedback system of ODE's appearing to exhibit linearly stable subharmonic orbits is

(10)

$$
\begin{array}{lll}
\frac{d u_{1}}{d t}=\beta u_{4}+\alpha(1+\cos t)^{m} u_{1}\left(1-u_{1}^{2}\right), & \frac{d u_{2}}{d t}=\beta u_{1}+\alpha(1+\cos t)^{m} u_{2}\left(1-u_{2}^{2}\right), \\
\frac{d u_{3}}{d t}=\beta u_{2}+\alpha(1+\cos t)^{m} u_{3}\left(1-u_{3}^{2}\right), & \frac{d u_{4}}{d t}=\beta u_{3}+\alpha(1+\cos t)^{m} u_{4}\left(1-u_{4}^{2}\right),
\end{array}
$$

for $t \in \mathbb{R}_{+}$, with an initial value $u(0)=u_{0} \in \mathbb{R}^{4}$, where $\alpha, \beta, m \in(0, \infty)$ are suitable constants. For instance, choosing $\alpha=\beta=1$ and $m=3$ one obtains (by numerical computations using a fourth-order Runge-Kutta method) a linearly stable $(8 \times 2 \pi)$-periodic orbit whose initial value at $t=0$ is approximately $u_{0}=(0.8906,1.0187,-0.8906,-1.0187)$. We do not have a formal analytic proof of this claim.

Observe that each of the systems (1), (5), and (10) can be written as an abstract evolution equation

$$
d u / d t=\mathscr{A} u+\mathscr{F}(t, u(t)), \quad t>0,
$$

where $\mathscr{A}: X \rightarrow X$ is the infinitesimal generator of a $C_{0}$-semigroup $\left\{e^{\mathscr{A} t}\right.$ : $\left.t \in \mathbb{R}_{+}\right\}$of strongly positive (bounded linear) operators in $X$, i.e., $e^{\mathscr{A} t} x \gg 0$ whenever $x>0$ in $X$ and $t \in(0, \infty)$, and $\mathscr{F}(t, \cdot): X \rightarrow X$ is a Nemytskii (substitution) operator in a function space $X$. We would like to point out that all three systems (1), (5), and (10) have the following common feature: The spectrum $\sigma(\mathscr{A})$ of $\mathscr{A}$ has the form $\sigma(\mathscr{A})=\left\{\lambda_{k} \pm i \mu_{k}: k=1,2, \ldots\right\}$ where $\lambda_{k}, \mu_{k} \in \mathbb{R}$ satisfy $\lambda_{1}>\lambda_{2} \geq \lambda_{3} \geq \cdots$ and $\mu_{1}=0<\mu_{2}$. Consequently, there exists $\lambda \in \mathbb{R}$ (e.g., $\lambda=\lambda_{2}$ ) such that the strongly monotone linear autonomous system

$$
d u / d t=\mathscr{A} u-\lambda u, \quad t>0,
$$

possesses a (linearly unstable) periodic orbit. In our examples above (cf. (11)) we have added the nonlinearity $\mathscr{F}(t, u(t))+\lambda u$ to the right-hand side of (12) 
in order to "stabilize" this unstable orbit. It would be interesting to find out whether linearly stable subharmonic orbits are caused by complex eigenvalues of $\mathscr{A}$ or, perhaps, by those of $\mathscr{A}+\mathscr{M}$ where $\mathscr{M}$ is any bounded pointwise multiplication operator in the function space $X$. Neither of these two possibilities can occur if $\mathscr{A}$ (and consequently $\mathscr{A}+\mathscr{M}$ ) is essentially selfadjoint in a suitable (real) Hilbert space $H$, such as $L^{2} \omega C(\mathbb{R} / 2 \pi \mathbb{Z})=X$ or $\mathbb{R}^{N}=X$.

As far as applications of our examples are concerned, time-periodic dynamical systems, when compared to their autonomous simplifications that do not exhibit any linearly stable periodic orbits, seem to be far more suitable for mathematical modelling of strongly monotone processes with regular (but strong) time-periodic seasonal variations.

\section{REFERENCES}

1. N. D. Alikakos and P. Hess, On stabilization of discrete monotone dynamical systems, Israel J. Math. 59 (1987), 185-194.

2. N. D. Alikakos, P. Hess, and H. Matano, Discrete order preserving semigroups and stability for periodic parabolic differential equations, J. Differential Equations 82 (1989), 322-341.

3. H. Amann, Existence and regularity for semilinear parabolic evolution equations, Ann. Scuola Norm. Sup. Pisa (4) 11 (1984), 593-676.

4. 47-83.

5. X.-Y. Chen and H. Matano, Convergence, asymptotic periodicity, and finite-point blow-up in one-dimensional semilinear heat equations, J. Differential Equations 78 (1989), 160-190.

6. E. N. Dancer and P. Hess, Stability of fixed points for order-preserving discrete-time dynamical systems, J. Reine Angew. Math. 419 (1991) 125-139.

7. J. K. Hale, Asymptotic behavior of dissipative systems, Math. Surveys Monographs, vol. 25, Amer. Math. Soc., Providence, RI, 1988.

8. J. K. Hale and A. S. Somolinos, Competition for fluctuating nutrient, J. Math. Biology 18 (1983), 255-280.

9. P. Hess and H. F. Weinberger, Convergence to spatial-temporal clines in the Fisher equation with time-periodic fitnesses, J. Math. Biology 28 (1990), 83-98.

10. M. W. Hirsch, Differential equations and convergence almost everywhere in strongly monotone semiflows, Contemp. Math., vol. 17, Amer. Math. Soc., Providence, R.I., 1983, pp. 267-285.

11. __ The dynamical systems approach to differential equations, Bull. Amer. Math. Soc. 11 (1984), 1-64.

12. __ Attractors for discrete-time monotone dynamical systems in strongly ordered spaces, Geometry and Topology, Lecture Notes in Math., vol. 1167, Springer-Verlag, Berlin, Heidelberg, and New York, 1985, pp. 141-153.

13. Stability and convergence in strongly monotone dynamical systems, J. Reine Angew. Math. 383 (1988), 1-53.

14. N. N. Lebedev, Special functions and their applications (transl. by R. A. Silverman), PrenticeHall, Englewood Cliffs, NJ, 1965.

15. H. Matano, Asymptotic behavior of solutions of semilinear heat equations on $S^{1}$, Nonlinear Diffusion Equations and Their Equilibrium States II, Math. Sci. Res. Inst. Publ., vol. 13, Springer-Verlag, New York, Berlin, and Heidelberg, 1988, pp. 139-162.

16. R. D. Nussbaum, Positive operators and elliptic eigenvalue problems, Math. Z. 186 (1984), 247-264.

17. P. Poláčik, Convergence in smooth strongly monotone flows defined by semilinear parabolic equations, J. Differential Equations 79 (1989), 89-110. 
18. J. F. Selgrade, Asymptotic behavior of solutions to single loop positive feedback systems, J. Differential Equations 38 (1980), 80-103.

19. H. L. Smith, Cooperative systems of differential equations with concave nonlinearities, Nonlinear Anal. 10 (1986), 1037-1052.

20. __ Systems of ordinary differential equations which generate an order preserving flow. A survey of results, SIAM Review 30 (1988), 87-113.

21. H. L. Smith and H. R. Thieme, Quasiconvergence and stability for strongly order-preserving semiflows, SIAM J. Math. Anal. 21 (1990), 673-692.

22. _ Convergence for strongly order-preserving semiflows, SIAM J. Math. Anal. 22 (1991), 1081-1101.

23. P. Takáč, Convergence to equilibrium on invariant d-hypersurfaces for strongly increasing discrete-time semigroups, J. Math. Anal. Appl. 148 (1990), 223-244.

24. __ Asymptotic behavior of discrete-time semigroups of sublinear, strongly increasing mappings with applications in biology, Nonlinear Anal. 14 (1990), 35-42.

25. _ Domains of attraction of generic $\omega$-limit sets for strongly monotone semiflows, $\mathrm{Z}$. Anal. Anwendungen 10 (1991), 275-317.

26. __ Domains of attraction of generic $\omega$-limit sets for strongly monotone discrete-time semigroups, J. Reine Angew. Math. 423 (1992), 101-173.

27. __ Asymptotic behavior of strongly monotone time-periodic dynamical processes with symmetry, J. Differential Equations (to appear).

Mathematics Department, Vanderbilt University, NashVille, Tennessee 37240

E-mail address: ptakac@athena.cas.vanderbilt.edu 